\title{
HAIR LOSS AFTER TRANSESPHENOIDAL SURGERY IN PATIENT WITH CURED ACROMEGALY
}

\author{
M. Sierra Bracamonte, M. Calatayud Gutiérrez, G. Allo Miguel, F. Hawkins Carranza. \\ Endocrinology and Nutrition Department. Doce de Octubre University Hospital. Madrid.
}

INTRODUCTION: Hair loss has been reported as an adverse effect of somatostatin analog therapy; on the other hand, hair loss after surgical treatment for acromegaly has been rarely described. In either case, the mechanism is still unknown. We report a case of a patient with acromegaly that presented with hair loss after surgical treatment

CASE DESCRIPTION: We describe a case of a 73 y.o female that was being studied for acromegaly because of enlarged facial features (Figure 1a). Laboratory results confirmed acromegaly: IGF-1 $875.2 \mathrm{ng} / \mathrm{ml}, \mathrm{GH} 16,20 \mathrm{ng} / \mathrm{ml}$, GH 120' postOGTT $54 \mathrm{ng} / \mathrm{ml}$.and the MRI showed a pituitary macroadenoma with suprasellar extension without sinus invasion or optic chiasm compression.

Transsphenoidal surgery was performed with complete tumor resection and no immediate complications. Postoperative IGF $-1, \mathrm{GH}$ and OGTT for $\mathrm{GH}$ were measured at 12 weeks confirming biochemical remission. The rest of the pituitary axis remained within normal range. Four months after surgery the patient presented with diffuse scalp hair loss, predominantly in the frontoparietal area, and complete loss of eyebrows (Figure 1b). She was diagnosed of a telogen effluvium. We performed a basic laboratory evaluation, which ruled out other etiologies (including hormonal disturbances) than might cause hair loss. Three months after the initial evaluation, she presented a spontaneous mild recovery in the frontal parietal area, with no signs of recovery in her eyebrows.

Figure 1. Photos of the patient at diagnosis (1a) and after hair loss (4 months after surgery) (1b).

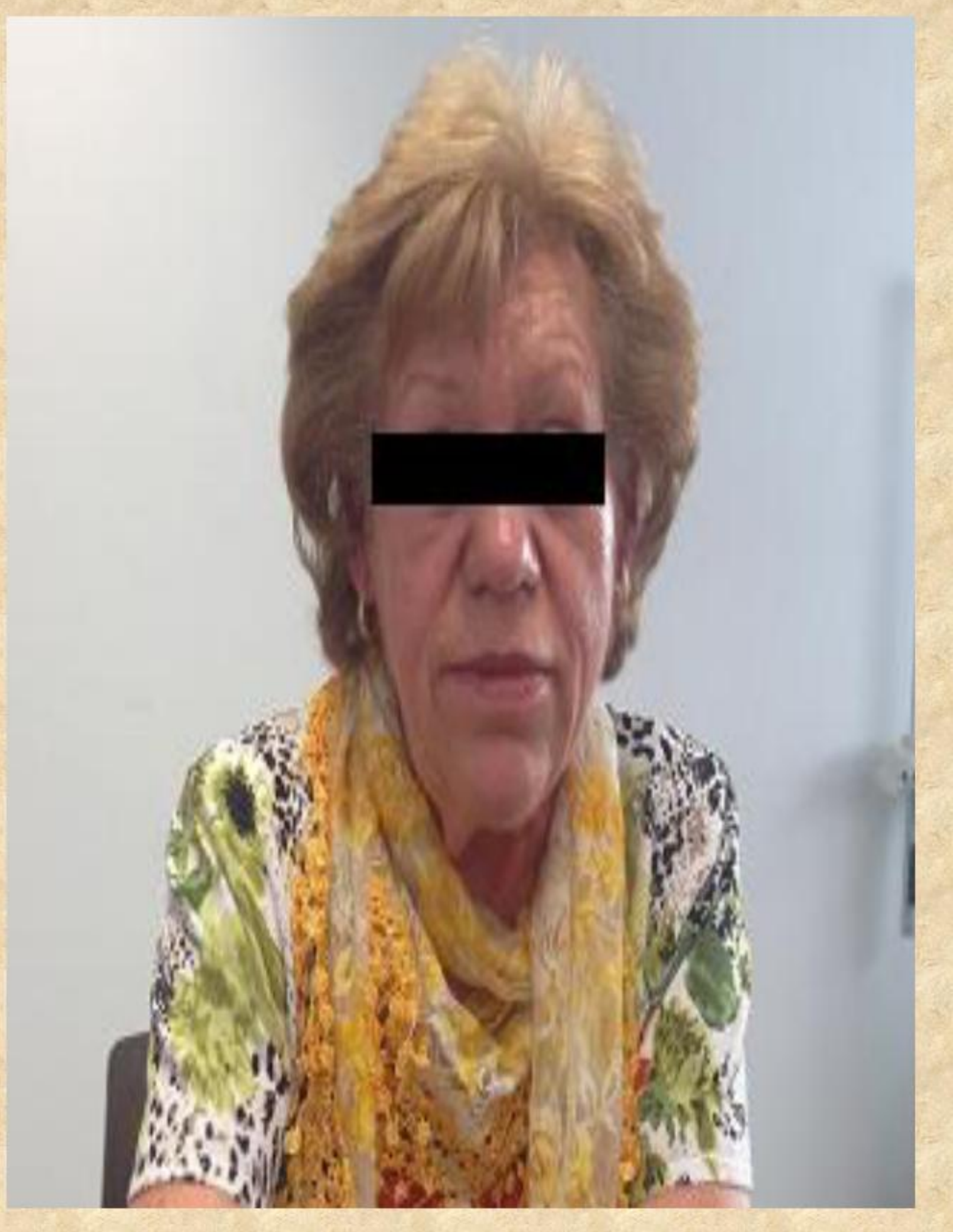

Fig.1a

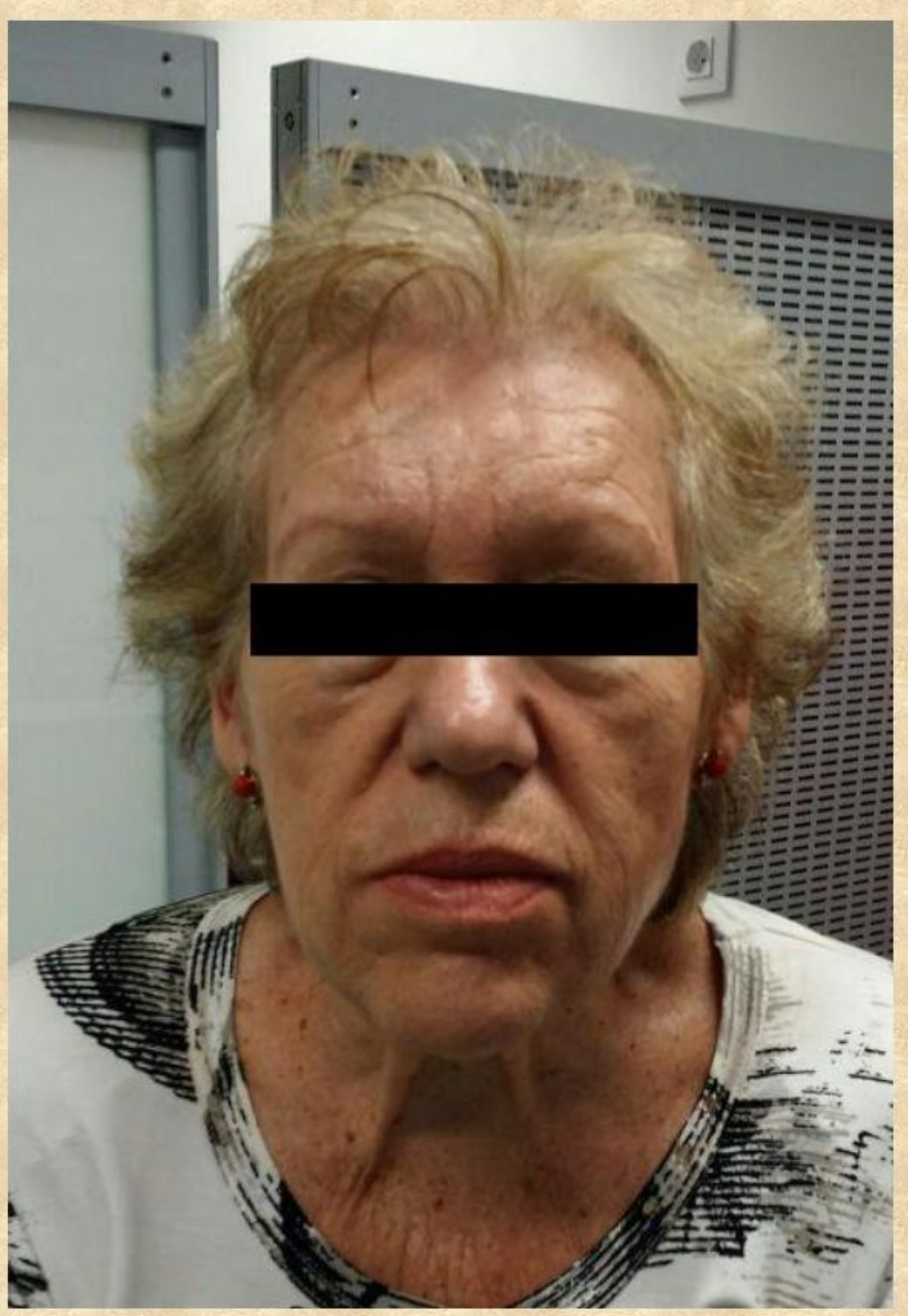

Fig.1b

DISCUSSION: Transsphenoidal surgery is the primary therapy in most acromegalic patients. Only one study has been published that describes hair loss after surgical treatment for acromegaly (1). In this study by Yamada et al telogen effluvium was observed approximately in $50 \%$ of patients who underwent surgery for acromegaly. Hair loss occurred generally in the first six months after surgery, mainly in women and patients that presented biochemical criteria for complete remission. The degree was generally mild, and the most common area of hair loss was the parietal region. In regard to recovery, full recovery was seen in $32 \%$, mostly women. Incomplete recovery was observed mainly in men and patients with severe scalp hair loss.

IGF-1 may play an important roll in the development and preservation of the hair follicle. The drastic descent of the IGF-1 levels could be implicated on the mechanism of telogen effluvium in these patients. The same mechanism has been suggested for the hair loss experienced in some patients treated with somatostin analogs and pegvisomat.

CONCLUSION: Hair loss can appear after transsphenoidal surgery for acromegaly. This condition may be more frequent than what has been reported, and might go unnoticed by clinicians or the patient himself. Questioning patients about hair loss could help us make an early diagnosis, understand its natural history, true frequency and risk factors. Finally, because hair loss has been associated with low self-esteem and quality of life improve in the knowledge of this entity could help clinicians to inform acromegalic patients about this possible outcomes, and the probably of recovery.

1. Yamada, S., N. Fukuhara, H. Nishioka, M. Yamaguchi- Okada, A. Takeshita, and Y. Takeuchi. (2013) Scalp hair loss after transsphenoidal adenomectomy in patients with acromegaly. Clin. Endocrinol. 79:386-393. 